\title{
Colour Change Sensitivity of Dye Recipes
}

\section{Document Version}

Accepted author manuscript

Link to publication record in Manchester Research Explorer

\section{Citation for published version (APA):}

Oulton, D., \& Chen, P. (1995). Colour Change Sensitivity of Dye Recipes. Society of Dyers and Colourists. Journal $, 111,237-244$.

\section{Published in:}

Society of Dyers and Colourists. Journal

\section{Citing this paper}

Please note that where the full-text provided on Manchester Research Explorer is the Author Accepted Manuscript or Proof version this may differ from the final Published version. If citing, it is advised that you check and use the publisher's definitive version.

\section{General rights}

Copyright and moral rights for the publications made accessible in the Research Explorer are retained by the authors and/or other copyright owners and it is a condition of accessing publications that users recognise and abide by the legal requirements associated with these rights.

\section{Takedown policy}

If you believe that this document breaches copyright please refer to the University of Manchester's Takedown Procedures [http://man.ac.uk/04Y6Bo] or contact uml.scholarlycommunications@manchester.ac.uk providing relevant details, so we can investigate your claim.

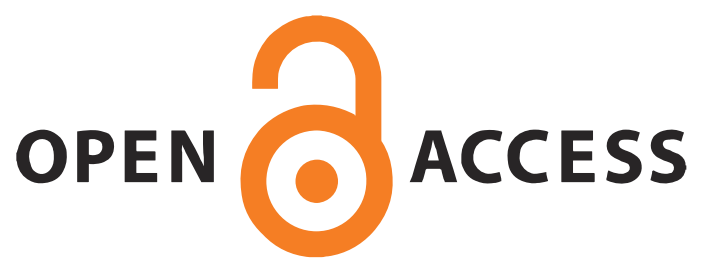




\title{
Colour change sensitivity of dye recipes
}

\author{
D P Oulton and P Chen
}

\author{
Dept of Textiles, UMIST, PO Box 88, Manchester M60 1QD, UK
}

\begin{abstract}
Relationships are investigated between concentration changes in multi-dye recipes and colour changes. Two methods are examined for predicting colour changes associated with small changes in dye concentration around ten colour centres. A synthetic reflectance curve generator and commercial match prediction system are used to generate the recipes. This simulates a dyeing process of zero variability and isolates the variables associated with the dyes. A matrix method is introduced to calculate $\Delta E$ for a given recipe concentration change in one or more dyes, possibly moving in opposite directions. The results are related to dye standardisations of $\pm 2.5 \%, 5 \%$ and $10 \%$ strength. The matrix method is confirmed using a manual match prediction program.
\end{abstract}

\section{INTRODUCTION}

A reproducible colour appearance and a high probability of being 'right first time' in a coloration process are always major objectives of dyers and colourists. The penalties for not achieving right-first-time processing in coloration are severe [1]. It has been reported that weighing and measuring errors are major sources of off-shade dyeings $[2,3]$. Inaccuracy of colorant dispensing is one of the major problems in the coloration process, especially in blind dyeing. The present investigation is concerned with the relationship of small colour changes and the recipe changes associated with them. The investigation also relates colorant standardisation to colour changes.

A model system has been established to isolate the variables concerned. The coloration process itself is assumed to be constant. In other words, the same recipe always produces an identical colour.

The model uses a $C M C(2: 1) \Delta E$ value of one to define a small volume of colour space in which to establish relationships between changes in colour and concentration. This in turn establishes the smallest recipe change that will produce a given colour difference. Changes along the semi-axes are analysed, i.e. chroma differences when hue and lightmess differences are zero, with equivalent pure hue and pure lightness differences. We reported in a previous paper that $\Delta E=1$ hue change results from much smaller recipe changes than $\Delta E=1$ lightness or chroma changes [4]. In this further investigation our aim was:

(a) To establish the relationship between small colour changes and recipe changes

(b) To relate the colour change sensitivity to a colorant standardisation limit [5].

In each case the results refer to the model system described above. This includes just one set of variables, i.e. colorant concentrations, which may affect colour uncertainty. All other variables were assumed constant. In a real coloration process additional sources of uncertainty in colour would be present.

\section{EXPERIMENTAL}

The UMIST Shademaster and Macbeth colour match prediction system were used for the generation of experimental data $[6,7]$.

\section{Generation of colour difference pairs}

The Shademaster system was designed to allow change of chroma, hue and lightness independently. Thus a colour $\mathrm{A}$ was selected, on Shademaster as a target colour in CIE colour space, then it was changed to colour $\mathrm{A}^{\prime}$ making the difference $\Delta E$ equal to 1 . This gives a pair of colours precisely one $\Delta E$ unit apart, with a specific direction of change, e.g. in hue only. Synthetic reflectance curves for these colours were then generated by the Shademaster system. The curves were generated using the $\mathrm{CMC}(2: 1)$ colour-difference formula. Three different reflectance curves were generated for samples changed in the hue, chroma and lightness directions from the target colour to $\Delta E=1$. The data from these reflectance curves were entered into the Matchmaker system to make recipe predictions. Pairs of reflectance curves representing colours one $\triangle E C M C(2: 1)$ unit apart were generated.

Table 1 Colorants (all BASF) used with Matchmaker

\begin{tabular}{ll}
\hline No. & Colorant name \\
\hline N2 & Helizarin Yellow G Conc \\
N3 & Helizarin Brilliant Yellow RRT Conc \\
N4 & Helizarin Brilliant Orange GT Conc \\
N5 & Helizarin Scartet B Conc \\
N6 & Helizarin Brilliant Red BBT \\
N7 & Helizarin Brilliant Violet BT \\
N8 & Helizarin Blue RT \\
N9 & Helizarin Blue Conc \\
N10 & Helizarin Green BT Conc \\
N11 & Helizarin Brown RRT Conc \\
N12 & Helizarin Dark Brown TT \\
N13 & Helizarin Black HDT \\
\hline
\end{tabular}


Table 2 Target shades, recipes and alterations for one $\Delta E$ unit change in lightness, chroma and hue

\begin{tabular}{|c|c|c|c|c|c|c|c|c|c|c|c|c|c|}
\hline Target & $\begin{array}{l}\text { Recipe } \\
\text { no. }\end{array}$ & Colorant & Conen & $c_{\mathrm{T}}$ & $C_{\mathrm{ch}}$ & $c_{n}$ & Target & $\begin{array}{l}\text { Recipe } \\
\text { no. }\end{array}$ & Cotorant & Concn & $G_{1}$ & $C_{\mathrm{eh}}$ & $c_{\mathrm{h}}$ \\
\hline \multirow[t]{9}{*}{ A } & \multirow[t]{3}{*}{1} & N2 & 0.7216 & -0.0652 & 0.1065 & 0.0005 & \multirow[t]{6}{*}{$F$} & \multirow[t]{3}{*}{11} & N2 & 2.566 & -0.6388 & 0.5608 & -0.2471 \\
\hline & & N5 & 0.0723 & -0.0089 & -0.0004 & -0.0006 & & & N4 & 0.034 & -0.0077 & -0.008 & -0.0042 \\
\hline & & N10 & 0.0283 & -0.0047 & -0.0005 & 0.0028 & & & Nto & 0.3111 & -0.0583 & 0.0154 & 0.0166 \\
\hline & \multirow[t]{3}{*}{2} & N3 & 0.2781 & -0.0253 & 0.0374 & -0.0008 & & \multirow[t]{3}{*}{12} & N2 & 2.717 & -0.6801 & 0.5592 & -0.2764 \\
\hline & & N5 & 0.0412 & -0.0054 & -0.0021 & -0.0025 & & & N6 & 0.0236 & -0.0056 & -0.0054 & -0.0025 \\
\hline & & N10 & 0.0397 & -0.0057 & 0.0001 & 0.0035 & & & N10 & 0.2795 & -0.0499 & 0.0216 & 0.0186 \\
\hline & \multirow[t]{3}{*}{3} & N2 & 0.7648 & -0.0711 & 0.1101 & -0.0009 & \multirow[t]{6}{*}{$\mathbf{G}$} & \multirow[t]{3}{*}{13} & N3 & 0.4969 & -0.2145 & -0.372 & -0.2242 \\
\hline & & N6 & 0.0607 & -0.0068 & -0.0005 & -0.0021 & & & N6 & 6.7554 & -0.9495 & 1.2205 & 0.1708 \\
\hline & & N10 & 0.021 & -0.0038 & -0.0003 & 0.0026 & & & N7 & 0.0527 & -0.0128 & -0.0369 & -0.0046 \\
\hline \multirow[t]{3}{*}{ B } & \multirow[t]{3}{*}{4} & N3 & 1.4692 & -0.1584 & 0.3609 & 0.0172 & & \multirow[t]{3}{*}{14} & N4 & 1.4876 & -0.4964 & -0.9966 & -0.6048 \\
\hline & & N5 & 0.4926 & -0.0388 & 0.0115 & -0.0643 & & & N6 & 5.9308 & -0.8726 & 1.7264 & 0.4407 \\
\hline & & N10 & 0.0344 & -0.0045 & -0.0038 & -0.0018 & & & N7 & 0.0681 & -0.0147 & -0.0473 & -0.0098 \\
\hline \multirow[t]{6}{*}{ c } & \multirow[t]{3}{*}{5} & N2 & 2.891 & -0.6936 & 0.6534 & -0.0649 & \multirow[t]{6}{*}{$\mathbf{H}$} & \multirow[t]{3}{*}{15} & N3 & 0.3962 & -0.0487 & 0.1249 & 0.0918 \\
\hline & & N10 & 0.1715 & -0.0274 & 0.0048 & 0.0157 & & & N4 & 2.0323 & -0.4386 & 0.1556 & -0.0858 \\
\hline & & N11 & 0.4903 & -0.066 & -0.0093 & -0.0137 & & & N10 & 0.0681 & -0.0137 & -0.005 & 0.0025 \\
\hline & \multirow[t]{3}{*}{6} & N3 & 1.1952 & -0.3016 & 0.2231 & -0.0252 & & \multirow[t]{3}{*}{16} & N2 & 4.101 & -1.4457 & 0.4586 & 0.3179 \\
\hline & & N10 & 0.2698 & -0.0487 & 0.0137 & 0.0208 & & & N4 & 1.0799 & -0.1511 & 0.1301 & -0.0536 \\
\hline & & N1t & 0.303 & -0.034 & -0.023 & $-0,0186$ & & & N7 & 0.0884 & -0.0176 & -0.005 & 0.002 \\
\hline \multirow[t]{6}{*}{ D } & \multirow[t]{3}{*}{7} & N5 & 0.2085 & -0.0345 & -0.0471 & 0.0156 & \multirow[t]{6}{*}{1} & \multirow[t]{3}{*}{17} & N3 & 2.6797 & -0.6775 & 0.4992 & 0.292 \\
\hline & & N7 & 0.2662 & -0.0297 & 0.0682 & 0.0103 & & & N5 & 1.1091 & -0.2 & 0.0625 & -0.0419 \\
\hline & & NB & 0.089 & -0.0184 & -0.0483 & -0.0218 & & & N10 & 0.0133 & -0.0047 & -0.0038 & 0.0019 \\
\hline & \multirow[t]{3}{*}{8} & NG & 0.3129 & -0.0511 & -0.0843 & 0,0243 & & \multirow[t]{3}{*}{18} & N2 & 7.1022 & -1.156 & 0.8906 & 0.5803 \\
\hline & & N7 & 0.1753 & -0.0232 & -0.089 & 0.0059 & & & N5 & 1.516 & -0.3507 & 0.1421 & -0.0557 \\
\hline & & NB & 0.1277 & -0.0209 & 0.1293 & -0.0197 & & & N10 & 0.0096 & -0.004 & -0.0031 & 0.0013 \\
\hline \multirow[t]{6}{*}{$\mathbf{E}$} & \multirow[t]{3}{*}{9} & N2 & 1.3091 & -0.2552 & 0.3305 & -0.0649 & & \multirow[t]{3}{*}{19} & N3 & 2.62 & -0.6634 & 0.511 & 0,2809 \\
\hline & & N7 & 0.0465 & -0.0098 & -0.0028 & -0.0019 & & & N5 & 0.9832 & -0.1588 & 0.0949 & -0.0629 \\
\hline & & N10 & 0.0911 & -0.0164 & 0.0074 & 0.0097 & & & N11 & 0.1446 & -0.0473 & -0.0379 & 0.0194 \\
\hline & 10 & N3 & 0.4916 & -0.1062 & 0.0762 & -0.03 & $J$ & 20 & N2 & 6.2769 & -1.1944 & 0.8453 & 0.3545 \\
\hline & & N7 & 0.0125 & -0.0035 & -0.0038 & -0.0014 & & & N4 & 0.5827 & -0.0655 & 0.0625 & -0.045 \\
\hline & & N10 & 0.1678 & -0.0321 & 0.0139 & 0.0126 & & & N7 & 0.0936 & -0.019 & -0.0045 & 0.003 \\
\hline
\end{tabular}

\section{Recipe prediction}

Standard recipe prediction procedures were used to generate recipes using a Macbeth Matchmaker system. There are twelve colorants available in the system, listed in Table 1. Three-colorant recipes were predicted. Typical results of the base recipes are listed in Table 2 .

The resulting recipes were analysed and compared for critical variables that might have affected colorant standardisation and colorant dispensing accuracy. All had a predicted $\Delta E<0.05$ to the input curve under illuminant $\mathrm{D}_{65}$, although metamerism was allowed under other illuminants.

\section{Colour variation with colorant concentration}

To reveal the variation of recipe $\Delta E, \Delta L, \Delta C$ and $\Delta H$ (where $\Delta L, \Delta C$ and $\Delta H$ are the lightness, chroma and hue changes) with colorant concentration, a second experiment was carried out. For a given target, a predicted recipe can be generated using the Matchmaker system. If a colorant concentration in this recipe is changed, the resulting colour will shift from the target. For a given concentration change of a colorant, there is a corresponding $\Delta E, \Delta L, \Delta C$ and $\Delta H$. The manual match program allows specific colorant concentration changes to be explored. This experiment was carried out for two targets with concentration changes of each colorant in increasing $1 \%$ steps until $\Delta E=1$ was reached. The plots of $\Delta E, \Delta L, \Delta C$ and $\Delta H$ versus $\Delta c$ are shown in Figure 1 (where $\Delta c$ is change in colorant concentration). This shows the effect of changing a single components of the three-colorant recipe.

It has been found that rate of change of colour with concentration varies substantially, thus the $x$-axis and $y$ axis scales in Figure 1 are varied. The slopes for colorant 1 are twice as steep as those for colorant 3 in this recipe. It follows that in this recipe colorant 1 is the major contributor to colour change sensitivity.

\section{RESULTS AND DISCUSSION}

Determining recipe sensitivity

In general, a CMC unit hue change corresponds to the subjectively smallest curve changes, while CMC unit lightness changes result in largest curve shifts. From Figure 1, it can be seen that there are nearly linear relationships between colorant concentration change and colour within a small volume of colour space, e.g. $\Delta E<1$.

If a linear relationship is assumed for all nine variables (three colorants and three dimensions of colour), it is possible to construct a set of nine equations linking concentration change to colour change in a given recipe. $R_{\text {, }}$ $R_{\mathrm{ch}}$ and $R_{\mathrm{h}}$ are defined as lightness, chroma and hue unit rates of change for unit colorant concentration change, i.e. $R_{1}=\Delta L / \Delta c, R_{\mathrm{ch}}=\Delta C / \Delta c$ and $R_{\mathrm{h}}=\Delta H / \Delta c($ Eqn 1$)$;

$$
\Delta L=\Delta c R_{\mathrm{l}} \quad \Delta C=\Delta c R_{\mathrm{ch}} \quad \Delta H=\Delta c R_{\mathrm{h}}
$$



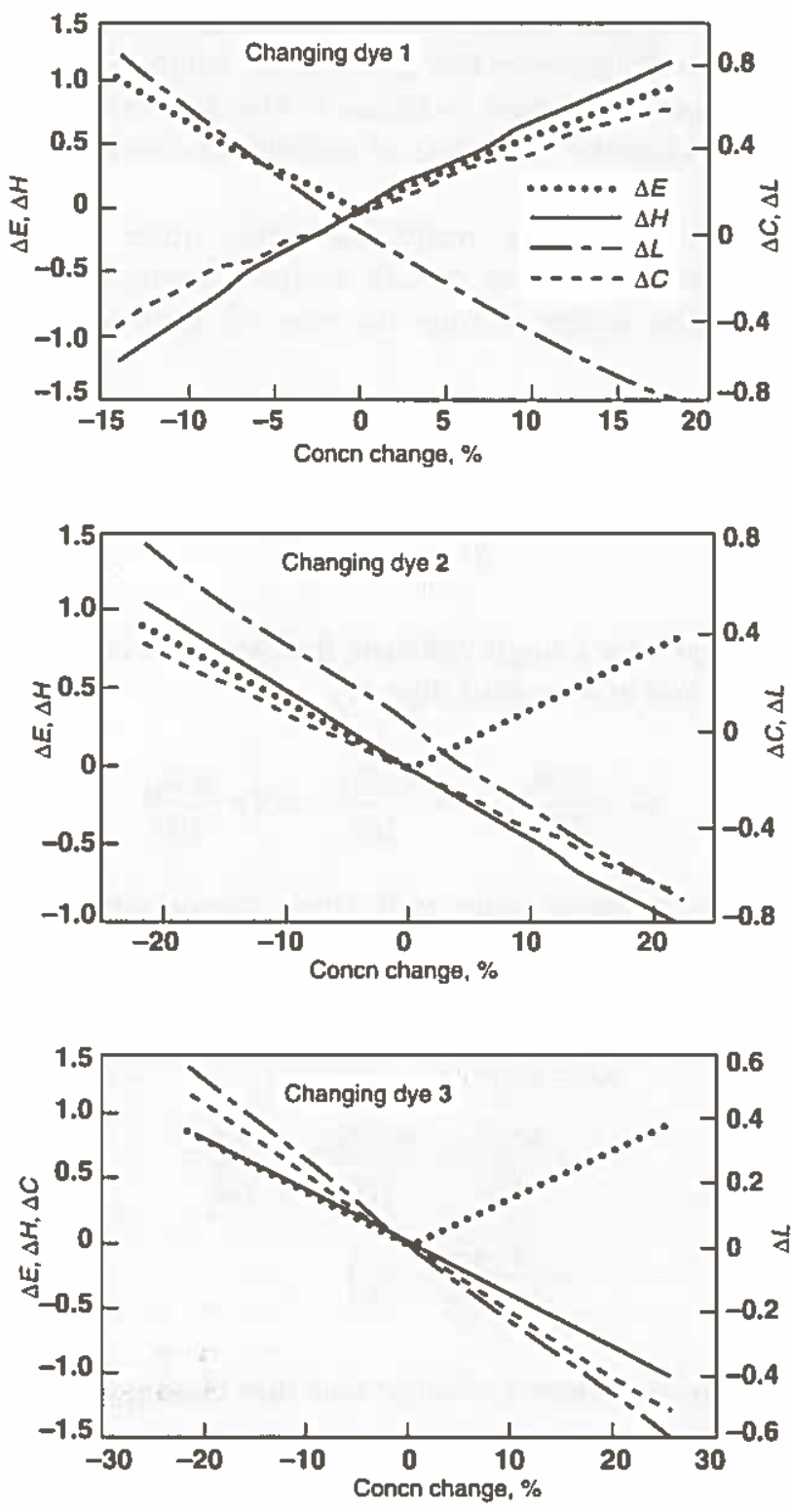

Figure 1 Target change corresponding dye concentration change

$R_{\mathrm{l}}, R_{\mathrm{ch}}$ and $R_{\mathrm{h}}$ are characteristic constant rates of change for a given colorant in a given recipe, expressing the linear relationships.

Using the values from Table 2 giving the concentration changes of colorants, for one $\Delta E$ unit change in lightness, chroma and hue separately, the values of $R_{\mathrm{l}}, R_{\mathrm{ch}}$ and $R_{\mathrm{h}}$ defined above can be calculated for each colorant.

It is now possible to establish the nine $R$ values $R_{11}$ to $R_{\mathrm{h} 3}$ in the matrix which relates the colorant concentration changes $\Delta c_{1}, \Delta c_{2}$ and $\Delta c_{3}$ to colour change $\Delta L, \Delta C$ and $\Delta H$, for all colorants in a given three-colorant recipe. In the following example, the three cases of lightness, chroma and hue changes only are used to generate the full matrix.

Table 2 shows recipe changes for $\mathrm{CMC}$ unit changes in lightness $\left(\Delta c_{1}\right)$, chroma $\left(\Delta c_{\text {ch }}\right)$ and hue $\left(\Delta c_{h}\right)$. Three-colorant recipes are given for a target colour using different colorant combinations (column headed 'Colorant') at different concentrations $(c)$. In recipe 8 , target colour $D$, which has CIELAB coordinates $L^{*}=46.0, C^{*}=34.91$ and $H^{*}=314.34$, as an example, recipe changes are given in Table 3 for moving the target colour along each of the colour-difference component vectors in CIELAB space corresponding to one $\mathrm{CMC}$ unit of colour difference.

The values in the $\Delta c_{1}$ column in Table 2 are the concentration changes of colorants for $\mathrm{CMC}$ unit lightness change only. For the chosen recipe 8 , if colorant concentration changes are $\Delta c_{6}=-0.0511, \Delta c_{7}=-0.0232$ and $\Delta c_{8}=-0.0209$, they will result in a shade change of one CMC unit difference, with CIELAB changes $\Delta L=2.1$, $\Delta C=0$ and $\Delta H=0$. Thus the changes due to individual colorants add up to give Eqn 2:

$$
\begin{aligned}
& \Delta L=\Delta L_{6}+\Delta L_{7}+\Delta L_{8}=2.1 \\
& \Delta C=\Delta C_{6}+\Delta C_{7}+\Delta C_{8}=0 \\
& \Delta H=\Delta H_{6}+\Delta H_{7}+\Delta H_{8}=0
\end{aligned}
$$

where subscripts 6, 7 and 8 refer to colorants N6, N7 and N8, and $\Delta L$ etc., are those used in Eqn 1. These can be related to concentration by Eqn 1, giving Eqn 3:

$$
\begin{aligned}
& \Delta L=\Delta C_{6} * R_{\mathrm{b}}+\Delta C_{7} * R_{\mathrm{b}}+\Delta \mathrm{C}_{8} * R_{\mathrm{bb}}=2.1 \\
& \Delta \mathrm{C}=\Delta \mathrm{C}_{6} * R_{\mathrm{ch6}}+\Delta \mathrm{C}_{7} * R_{\mathrm{ch} 7}+\Delta \mathrm{C}_{8} * R_{\mathrm{ch} 8}=0 \\
& \Delta H=\Delta \mathrm{C}_{6}{ }^{*} R_{\mathrm{hb}}+\Delta \mathrm{C}_{7} * R_{\mathrm{h} 7}+\Delta \mathrm{C}_{8} * R_{\mathrm{hg}}=0
\end{aligned}
$$

Substituting the concentration change $\Delta c_{6}, \Delta c_{7}$ and $\Delta c_{8}$ with the appropriate values from Table $2(-0.0511,-0.0232$ and -0.0209; recipe 8, lightness change only) gives Eqn 4:

$$
\begin{aligned}
& -0.0511 R_{\mathrm{l}}-0.0232 R_{\mathrm{b}}-0.0209 R_{\mathrm{lg}}=2.1 \\
& -0.0511 R_{\mathrm{ch} 6}-0.0232 R_{\mathrm{ch} 7}-0.0209 R_{\mathrm{ch} 8}=0 \\
& -0.0511 R_{\mathrm{h} 6}-0.0232 R_{\mathrm{h} 7}-0.0209 R_{\mathrm{h} 8}=0
\end{aligned}
$$

If in the same way the columns $\Delta c_{\mathrm{ch}}$ and $\Delta c_{\mathrm{h}}$ (Table 2) are analysed, then for chroma change only (Eqn 5):

$$
\begin{aligned}
& -0.0843 R_{\mathrm{l} 6}-0.0890 R_{\mathrm{V}}+0.1293 R_{\mathrm{bs}}=0 \\
& -0.0843 R_{\mathrm{ch} 6}-0.0890 R_{\mathrm{ch} 7}+0.1293 R_{\mathrm{ch} 8}=2.2 \\
& -0.0843 R_{\mathrm{hb}}-0.0890 R_{\mathrm{h} 7}+0.1293 R_{\mathrm{h} 8}=0
\end{aligned}
$$

and for hue change only (Eq̨n 6):

$$
\begin{aligned}
& 0.0243 R_{\mathrm{b}}+0.0059 R_{\mathrm{b}}-0.0197 R_{\mathrm{lB}}=0 \\
& 0.0243 R_{\mathrm{ch6}}+0.0059 R_{\mathrm{ch} 7}-0.0197 R_{\mathrm{ch}}=0 \\
& 0.0243 R_{\mathrm{hb}}+0.0059 R_{\mathrm{h} 7}-0.0197 R_{\mathrm{h} 8}=1.45
\end{aligned}
$$

These nine equations can be expressed as a matrix equation (Eqn 7):

$$
\begin{aligned}
\left(\begin{array}{rrr}
-0.0511 & -0.0232 & -0.0209 \\
-0.0843 & -0.0890 & 0.1293 \\
0.0243 & 0.0059 & -0.0197
\end{array}\right)\left(\begin{array}{lll}
R_{\mathrm{l} 6} & R_{\mathrm{chb}} & R_{\mathrm{hb}} \\
R_{\mathrm{b}} & R_{\mathrm{ch} 7} & R_{\mathrm{h} 7} \\
R_{\mathrm{lB}} & R_{\mathrm{ch} 8} & R_{\mathrm{hB}}
\end{array}\right)_{(7)} \\
=\left(\begin{array}{ccc}
2.1 & 0 & 0 \\
0 & 2.2 & 0 \\
0 & 0 & 1.45
\end{array}\right)
\end{aligned}
$$


Table 3 Recipe changes for moving target colour along each colourdifference component vector in CIELAB space corresponding to one CMC unit of colour difference

\begin{tabular}{llll}
\hline Vector & $\Delta L^{*}$ & $\Delta C$ & $\Delta H^{*}$ \\
\hline Lightness only & 2.1 & 0 & 0 \\
Chroma only & 0 & 2.2 & 0 \\
Hue only & 0 & 0 & 1.45 \\
\hline
\end{tabular}

The solution of this matrix equation is Eqn 8:

$$
\left(\begin{array}{lll}
R_{\mathrm{l6}} & R_{\mathrm{ch} 6} & R_{\mathrm{h} 6} \\
R_{\mathrm{g}} & R_{\mathrm{ch} 7} & R_{\mathrm{h} 7} \\
R_{\mathrm{lB}} & R_{\mathrm{ch} 8} & R_{\mathrm{h} 8}
\end{array}\right)=\left(\begin{array}{ccc}
-17.36 & 10.66 & 58.83 \\
-25.97 & -27.82 & -101.3 \\
-29.20 & 4.817 & -31.38
\end{array}\right)
$$

The numeric subscripts indicating values for the individual colorants N6, N7 and N8.

The generalised form of Eqn 7 is the matrix equation (Eqn 9):

$$
\begin{array}{r}
\left(\begin{array}{lll}
\Delta C_{\mathrm{lt}} & \Delta C_{12} & \Delta C_{13} \\
\Delta C_{\mathrm{ch} 1} & \Delta C_{\mathrm{ch} 2} & \Delta C_{\mathrm{ch} 3} \\
\Delta C_{\mathrm{h} 1} & \Delta C_{\mathrm{h} 2} & \Delta C_{\mathrm{h} 3}
\end{array}\right)\left(\begin{array}{lll}
R_{\mathrm{l}} & R_{\mathrm{ch} 1} & R_{\mathrm{h} 1} \\
R_{\mathrm{l} 2} & R_{\mathrm{ch} 2} & R_{\mathrm{h} 2} \\
R_{13} & R_{\mathrm{ch} 3} & R_{\mathrm{h} 3}
\end{array}\right) \\
=\left(\begin{array}{ccc}
\Delta L^{*} & 0 & 0 \\
0 & \Delta C^{*} & 0 \\
0 & 0 & \Delta H^{*}
\end{array}\right)
\end{array}
$$

where $\Delta c$ is concentration change, subscripts 1,2 and 3 are the recipe components, subscripts $\mathrm{l}$, ch and $\mathrm{h}$ mean that the $\Delta v$ is taken for lightness change $\Delta L^{*}$, chroma change $\Delta C^{*}$ and hue change $\Delta H^{*}$ respectively. This makes it possible to establish the nine $R$ values $R_{11}$ to $R_{\mathrm{h} 3}$ in the matrix which relate the colorant concentration changes to colour change for all colorants in a given three-colorant recipe.

Table 4 shows the results of the application of Eqn 9 to Table $\mathbf{2}$ for six recipes. It contains the rates of change data for each colorant in each recipe. This assumes that rate of change is linear over the appropriate small volume of colour space, as shown in Figure 1. The data can now be used to examine the effect of individual concentration changes.

The variations in individual rates must now be combined to give an overall recipe sensitivity $\delta$, i.e. percentage recipe change for one $\Delta E$ unit of colour change.

For given $R_{\mathrm{l}}, R_{\mathrm{ch}}$ and $R_{\mathrm{h}}$ percentage concentration (c) change of each colorant the $\delta$ value is defined by Eqn 10:

$$
\delta=\frac{\Delta c}{c} \times 100
$$

From Eqn 1 for a single colorant, the changes in lightness, chroma and hue are thus (Eqn 11):

$$
\Delta L=\frac{\delta c R_{1}}{100}, \Delta C=\frac{\delta c R_{\mathrm{ch}}}{100}, \Delta H=\frac{\delta c R_{\mathrm{h}}}{100}
$$

Then, for a given recipe with three components, if the concentrations of three colorants are $c_{1}, c_{2}$ and $c_{3}$, the equations above become Eq̨n 12:

$$
\begin{aligned}
\Delta L & =\Delta L_{1}+\Delta L_{2}+\Delta L_{3} \\
& =\frac{\delta c_{1} R_{11}}{100}+\frac{\delta c_{2} R_{12}}{100}+\frac{\delta c_{3} R_{13}}{100} \\
& =\frac{\delta}{100} \sum_{i=1}^{3}\left(c_{i} R_{1 i}\right)
\end{aligned}
$$

\begin{tabular}{|c|c|c|c|c|c|c|c|c|c|}
\hline \multicolumn{4}{|c|}{1} & \multicolumn{3}{|l|}{2} & \multicolumn{3}{|l|}{3} \\
\hline Colorant & $R_{\mathrm{h}}$ & $R_{\mathrm{ch}}$ & $R_{1}$ & $R_{h}$ & $A_{\text {ch }}$ & $\boldsymbol{R}_{1}$ & $R_{h}$ & $R_{\mathrm{eh}}$ & $R_{1}$ \\
\hline $\begin{array}{l}1 \\
2 \\
3\end{array}$ & $\begin{array}{c}0.888376 \\
-183.828 \\
336.2866\end{array}$ & $\begin{array}{c}21.69844 \\
-100.868 \\
-97.5378\end{array}$ & $\begin{array}{l}-1.41385 \\
-174.365 \\
-161.657\end{array}$ & $\begin{array}{c}-12.4273 \\
-209.258 \\
253.404\end{array}$ & $\begin{array}{c}55.65785 \\
-156.329 \\
-98.9417\end{array}$ & $\begin{array}{l}-11.9207 \\
-219.911 \\
-159.804\end{array}$ & $\begin{array}{c}0.067111 \\
-210.781 \\
375.931\end{array}$ & $\begin{array}{l}20.78951 \\
-152.543 \\
-116.011\end{array}$ & $\begin{aligned} &-1.56367 \\
&-231.707 \\
&-187.689\end{aligned}$ \\
\hline & 20 & & & 7 & & & g & & \\
\hline Colorant & $R_{\mathrm{h}}$ & $R_{\text {ch }}$ & $R_{1}$ & $R_{h}$ & $R_{\text {en }}$ & $R_{1}$ & $R_{h}$ & $A_{c h}$ & $R_{1}$ \\
\hline $\begin{array}{l}1 \\
2 \\
3\end{array}$ & $\begin{aligned} & t .138436 \\
-16.4634 & -14.8101\end{aligned}$ & $\begin{array}{r}1.945582 \\
5.832675 \\
-142.413\end{array}$ & $\begin{array}{cc}2 & -0.02409 \\
5 & -6.63427 \\
-96.6676\end{array}$ & $\begin{array}{l}33.76113 \\
-10.0391 \\
-47.0977\end{array}$ & $\begin{array}{c}-15.9416 \\
19.7921 \\
-2.05646\end{array}$ & $\begin{array}{l}-17.9858 \\
-32.3667 \\
-28.1631\end{array}$ & $\begin{array}{c}58.83015 \\
-101.311 \\
-31.3787\end{array}$ & $\begin{array}{c}10.65911 \\
-27.8171 \\
4.817032\end{array}$ & $\begin{array}{l}-17.3641 \\
-25.9696 \\
-29.1963\end{array}$ \\
\hline
\end{tabular}

Similarly, for chroma change and hue change (Eqns 13 and 14):

$$
\begin{aligned}
& \Delta C=\frac{\delta}{100} \sum_{i=1}^{3}\left(c_{i} R_{\mathrm{ch} i}\right) \\
& \Delta H=\frac{\delta}{100} \sum_{i=1}^{3}\left(c_{i} R_{\mathrm{h} i}\right)
\end{aligned}
$$

Table 4 Ratios of colour change to colorant concentration change with different recipe numbers from Table 2 
The CMC(2:1) colour-difference formula is (Eqn 15):

$$
\Delta E=\left[\left(\frac{\Delta L^{*}}{2 S_{L}}\right)^{2}+\left(\frac{\Delta C^{*}}{S_{C}}\right)^{2}+\left(\frac{\Delta H^{*}}{S_{H}}\right)^{2}\right]^{1 / 2}
$$

Hence from Eqns 13-15 the percentage concentration change of each colorant may be calculated by substitution for $\Delta L, \Delta C$ and $\Delta H$ to give an overall recipe sensitivity $\delta$ (Eqn 16):

$$
\delta=\frac{100}{\left\{\left[\sum \frac{c_{i} R_{l i}}{2 S_{L}}\right]^{2}+\left[\sum \frac{c_{i} R_{\mathrm{chi}}}{S_{\mathrm{C}}}\right]^{2}+\left[\sum \frac{c_{i} R_{\mathrm{hi}}}{S_{H}}\right]^{2}\right\}^{1 / 2}}
$$

where the tolerance values $S_{L}, S_{C}$ and $S_{H}$ are those of the $\mathrm{CMC}(l: c)$ difference formula.

\section{Effects of sensitivity in various combinations}

The effect of the individual colorants reaching their tolerance limits is now examined. Table 5 shows the full permutation of possible recipe deviations, + indicating a maximum upward strength deviation, - indicating a downward maximum deviation. Permutations 21-26 show worst-case scenarios where individual colorants move to the limit in opposite directions. Fortunately Table 5 is symmetrical: +00 produces an effect equal and opposite to -00 (Figure 1 ), so only 13 variations are examined in Tables 6 and 7, i.e. 13 combinations. A fourcolorant recipe would have $3^{4}=81$ combinations.

For each recipe, nine simultaneous equations are solved (Eqn 9), giving the values illustrated in Table 4. For a chosen recipe and for each combination of concentration changes, a set of $\delta$ values are then derived using Eqn 16. Their column average expresses the overall sensitivity of the chosen recipe (Tables 6 and 7). Table 6 shows the sensitivities of these recipes, as percentage colorant concentration changes ( $\delta$ values). Table 7 shows these transformed into $\Delta E$ values, assuming $\pm 2.5 \%$ strength

Table 5 Full permutation of possible recipe deviations of individual

\begin{tabular}{|c|c|c|c|c|c|c|c|c|c|c|c|}
\hline & \multicolumn{3}{|c|}{ Recipe change } & \multicolumn{4}{|c|}{ Recipe change } & & \multicolumn{3}{|c|}{ Recipe change } \\
\hline & A & B & C & & A & B & C & & A & B & C \\
\hline 1 & + & 0 & 0 & 2 & - & 0 & 0 & 3 & 0 & + & 0 \\
\hline 4 & 0 & - & 0 & 5 & 0 & 0 & + & 6 & 0 & 0 & - \\
\hline 7 & 0 & + & + & 8 & 0 & - & - & 9 & 0 & + & - \\
\hline 10 & 0 & - & + & 11 & + & 0 & + & 12 & - & 0 & $\overline{-}$ \\
\hline 13 & + & 0 & - & 14 & - & 0 & + & 15 & + & + & 0 \\
\hline 16 & - & - & 0 & 17 & + & - & 0 & 18 & - & + & 0 \\
\hline 19 & + & + & + & 20 & - & - & - & 21 & + & + & - \\
\hline 22 & - & - & + & 23 & + & - & + & 24 & - & + & - \\
\hline 25 & - & + & + & 26 & + & - & - & 27 & 0 & 0 & 0 \\
\hline
\end{tabular}
dyes reaching their tolerance limits ${ }^{a}$

a $A, B$ and $C$ afe the recipe components

+ signifies a positive change of dye concentration - a negative change of dye concentration and 0 no change of dye concentration changes. Averaging along a row shows the effect of a given combination across all 20 recipes. Thus a row average indicates the severity of a given combination of changes.

\section{Recipe sensitivity}

From the results shown in Table 6 , it is clear that recipe sensitivities are different in different situations. For a given recipe, the sensitivity varies for different directions of change and combinations of direction. When concentrations of colorants are changed in the same direction, or just a single colorant concentration is changed, a small colour change may be expected (combinations 1-6 and 10). When colorants are changed in opposite directions, there may be a large change of colour (combinations 7-9 and 11-13).

In Table 6, combinations 1-3 involve an increase in strength of either colorant 1, 2 or 3 in the recipe. Column 1 shows that for this recipe the concentration of colorant 1 must be increased by $14.88 \%$ to give a colour change of one $\Delta E$ unit. Colorant 2 only requires a $9.18 \%$ change and colorant 3 requires a $14.38 \%$ change.

Recipe 8 is highly sensitive to combination 13 . If colorant 1 is $3.53 \%$ under strength and colorants 2 and 3 $3.53 \%$ over strength, a $\Delta E 1$ shade change results. By contrast, it would take a $49.37 \%$ increase in strength in both colorants 1 and 3 (the minor components) to produce a $\Delta E 1$ change in recipe 13 (combination 5). Combination 8 involves the same two colorants in the same recipe, this time moving in opposite directions. With this combination much smaller concentration changes $(\mathbf{1 4 . 7 1 \% )}$ ) cause a $\triangle E 1$ shift, for this recipe.

Selection of colorants in a recipe is thus quite important. For example, recipes 7 and 8 are two recipes for the same target colour. Their sensitivities are $17.45 \%$ and $11.13 \%$ (column average Table 6), indicating the latter is significantly more sensitive to dispensing or standardisation error. These values translate into $\Delta E$ values in Table 7 which shows a double $\Delta E$ uncertainty for recipe 8 against recipe 7 .

\section{Relating recipe sensitivity to colorant standardisation}

Since the results in Table 6 relate to a colour change of one $\Delta E$ unit, and the relationship between $\Delta E$ and $\Delta c$ is linear, it is possible to relate the recipe sensitivity to colorant standardisation. For a certain combination and a given $\delta$ value in Table 6 , colorant standardisation of $\pm 2.5 \%$ strength would be expected to produce $\Delta E(C M C)$ of $2.5 / \delta$. From Table 6, the results shown in Table 7 can be calculated. The effects of limiting colorant strength variation to $\pm 2.5 \%$ are clear. Even the worst-case scenario of combination 13, with all three colorants moving to their standardisation limits in opposite directions, produces few problems. However, recipes 8 (at $\Delta E 0.71$ ) and 20 at ( $\Delta E$ 0.47 ) might be troublesome.

It should, however, be emphasised that colorant strength variation is only one possible cause of colour uncertainty. 
Analysis of 260 recipes

Figure 2 gives a cumulative frequency analysis of Table 7 ( $\pm 2.5 \%$ strength variation), taking each recipe change combination as an independent case (260 recipes). Similarly, cumulative frequency analysis can be calculated from Table 6 at colorant standardisation values of $\pm 5 \%$ and $10 \%$; the results are shown in Figures 3 and 4.

Over all the 260 recipes in Table 7 , an average $\triangle E(C M C)$ uncertainty of $0.23,0.46$ and 0.92 (with colorant standardising limits of $\pm 2.5 \%, 5 \%$ and $10 \%$ respectively) is expected, due solely to colorant strength variation.

\section{Confirmation of calculated sensitivity by straight match prediction}

The prediction of sensitivity by the matrix method given above is based on $\Delta E$ changes of one unit in hue, chroma and lightness. It relies on a substantially linear relationship, over a small volume of colour space, between concentration change and colour change. It is possible, but laborious, to achieve the same result using a match prediction system. In this alternative approach, a given recipe is varied manually by, say, $\pm 2.5 \%$ in each component, and in varying combinations to produce results similar to those in Table 7.

The matrix method was used as a research tool to reveal the underlying characteristics of recipe sensitivity. The match prediction approach (based on systematic recipe variation by known percentage change) is more practicable for general use, as it does not depend on a specialised synthetic reflectance curve generator.

In order to confirm the validity of the results given by the matrix method of Eqns 9 and 16, match prediction was used. Thirteen combinations and two levels of difference (i.e. 26 variants of recipe 20 in Table 6, target colour J) were examined. Concentration changes predicted by the matrix method to produce $\Delta E$ values of 1.0 and 0.5 were used to

Table 6 Colorant concentration change $(\%)$ for $\Delta E=1^{\text {a }}$

\begin{tabular}{|c|c|c|c|c|c|c|c|c|c|c|c|c|c|c|}
\hline \multirow{2}{*}{$\begin{array}{l}\text { Colorant } \\
\text { combination }\end{array}$} & \multicolumn{3}{|c|}{ Colorant no. } & \multicolumn{11}{|c|}{ Recipe no. } \\
\hline & 1 & 2 & 3 & 1 & 2 & 3 & 4 & 5 & 6 & 7 & 8 & 9 & 10 & \\
\hline 1 & + & 0 & 0 & 14.88 & 13.98 & 14.68 & 24.24 & 24.26 & 17.92 & 18.47 & 7.49 & 22.38 & 14.78 & \\
\hline 2 & 0 & + & 0 & 9.18 & 13.4 & 8.97 & 14.4 & 11.96 & 11.1 & 19.43 & 7.89 & 17.59 & 40.95 & \\
\hline 3 & 0 & 0 & + & 14.38 & 13.16 & 17.26 & 16.87 & 9.64 & 14.12 & 31,84 & 30.19 & 13.43 & 11.81 & \\
\hline 4 & 0 & + & + & 12.04 & 13.39 & 10.87 & 13 & 10.38 & 9.79 & 13.29 & 6.37 & 14.55 & 11.8 & \\
\hline 5 & + & 0 & + & 10.85 & 12.99 & 12.16 & 12.33 & 9.95 & 10.99 & 25.36 & 8.85 & 17.63 & 20.94 & \\
\hline 6 & + & + & 0 & 9.22 & 9.61 & 9.07 & 15.16 & 13.42 & 17.99 & 14.86 & 20.2 & 13.42 & 13.74 & \\
\hline 7 & 0 & + & - & 6.14 & 7.64 & 6.58 & 9.64 & 6.17 & 7.95 & 24.95 & 10.15 & 8.83 & 10.94 & \\
\hline 8 & + & 0 & - & 9.9 & 7.94 & 10.41 & 16.12 & 8.32 & 11.2 & 12.62 & 6.32 & 9.18 & 6.86 & \\
\hline 9 & + & - & 0 & 6.9 & 9.73 & 6.74 & 10.72 & 9.2 & 7.19 & 12.28 & 3.91 & 14,28 & 14.07 & \\
\hline 10 & + & + & + & 12.96 & 13.07 & 12.03 & 13.06 & 13.41 & 13.9 & 14.35 & 19.14 & 20.38 & & \\
\hline 11 & + & + & - & 6.06 & 6.05 & 6.45 & 10.59 & 6.11 & 9.69 & 13.8 & 21.14 & 7.17 & 6.66 & \\
\hline 12 & + & - & + & 5.36 & 7.64 & 5.64 & 7.91 & 5.86 & 6.06 & 17.6 & 4.35 & 9.92 & 17.29 & \\
\hline 13 & - & + & + & 7.7 & 8.02 & 7.2 & 10.89 & 7.88 & 6.82 & 9.13 & 3.53 & 9.66 & 6.88 & \\
\hline \multirow[t]{2}{*}{$\bar{\sigma}$} & & & & 9.66 & 10.51 & 9.85 & 13.38 & 10.48 & 11.09 & 17.45 & 11.13 & 13.63 & 15.16 & \\
\hline & \multicolumn{3}{|c|}{ Dye no. } & \multicolumn{11}{|c|}{ Recipe no. } \\
\hline $\begin{array}{l}\text { Colorant } \\
\text { combination }\end{array}$ & 1 & 2 & 3 & 11 & 12 & 13 & 14 & 15 & 16 & 17 & 18 & 19 & 20 & $\bar{\sigma}$ row \\
\hline 1 & + & 0 & 0 & 21.96 & 21.41 & 35.85 & 26.61 & 34.77 & 11.33 & 19.49 & 20.28 & 27.37 & 12.83 & 20.25 \\
\hline 2 & 0 & + & 0 & 31.75 & 33.09 & 17.63 & 21.63 & 14.05 & 9.02 & 10.65 & 12.33 & 10.48 & 11.45 & 16.35 \\
\hline 3 & 0 & 0 & + & 12.18 & 12.85 & 23.99 & 19.56 & 14.47 & 9.51 & 41.98 & 41.49 & 32.7 & 15.53 & 19.85 \\
\hline 4 & 0 & + & + & 13.09 & 12.66 & 11.85 & 11.9 & 22.41 & 5.24 & 11.29 & 11.65 & 10.06 & 8.49 & 11.71 \\
\hline 5 & + & 0 & + & 17.86 & 19.18 & 49.37 & 44.11 & 12.09 & 18.94 & 17.21 & 20.29 & 21.85 & 15.47 & 18.92 \\
\hline 6 & + & + & 0 & 16.67 & 18.12 & 18.55 & 19.68 & 16.22 & 16.48 & 15.97 & 18.75 & 13.82 & 15.49 & 15.32 \\
\hline 7 & 0 & + & - & 10.19 & 11.4 & 18.95 & 20.26 & 7.52 & 9.88 & 9.57 & 11.99 & 9.91 & 10.17 & 10.94 \\
\hline 8 & + & 0 & $=$ & 8.31 & 8.53 & 14.71 & 11.52 & 15.15 & 5.35 & 18.17 & 16.67 & 20.22 & 7.84 & 11.27 \\
\hline 9 & + & - & 0 & 19.86 & 17.83 & 14.03 & +4.88 & 11.2 & 5.23 & 7.26 & 8.12 & 8 & 6.56 & 10.4 \\
\hline 10 & + & + & + & 19.03 & 18.72 & 14.57 & 15.81 & 22.49 & 9.21 & 17.89 & 18.11 & 13.09 & 15.1 & 15,42 \\
\hline 11 & + & + & - & 7.47 & 8.07 & 14.77 & 12.51 & 8.12 & 7.52 & 13.07 & 16.22 & 12.4 & 9.03 & 10.15 \\
\hline 12 & + & - & + & 13.49 & 15.06 & 19.83 & 27.71 & 6.77 & 7.38 & 6.86 & 8.17 & 7.76 & 7.21 & 10.39 \\
\hline 13 & - & + & + & 8.75 & 8.46 & 9.49 & 8.78 & 16.53 & 3.61 & 7.49 & 7.77 & 12.17 & 5.31 & 8.3 \\
\hline $\bar{\sigma}$ & & & & 15.43 & 16.8 & 20.28 & 19.61 & 15.52 & 9.13 & 15.14 & 16.3 & 15.37 & 10.81 & 13.84 \\
\hline
\end{tabular}

a Percentage dye concentration change is applied to each component (dyes 1-3) in the direction indicated 
Table $7 \Delta E$ values expected with colorant standardisation of $\pm 2.5 \%$ applied in the pattem indicated by each combination

\begin{tabular}{|c|c|c|c|c|c|c|c|c|c|c|c|}
\hline \multirow{2}{*}{$\begin{array}{l}\text { Colorant } \\
\text { combination }\end{array}$} & \multicolumn{11}{|c|}{ Recipe no. } \\
\hline & 1 & 2 & 3 & 4 & 5 & 6 & 7 & 8 & 9 & 10 & \\
\hline 1 & 0.17 & 0.18 & 0.17 & 0.10 & 0.10 & 0.14 & 0.14 & 0.33 & 0.11 & 0.17 & \\
\hline 2 & 0.27 & 0.19 & 0.28 & 0.17 & 0.21 & 0.23 & 0.13 & 0.32 & 0.14 & 0.06 & \\
\hline 3 & 0.17 & 0.19 & 0.14 & 0.15 & 0.26 & 0.18 & 0.08 & 0.08 & 0.19 & 0.21 & \\
\hline 4 & 0.21 & 0.19 & 0.23 & 0.19 & 0.24 & 0.26 & 0.19 & 0.39 & 0.17 & 0.21 & \\
\hline 5 & 0.23 & 0.19 & 0.21 & 0.20 & 0.25 & 0.23 & 0.10 & 0.28 & 0.14 & 0.12 & \\
\hline 6 & 0.27 & 0.26 & 0.28 & 0.16 & 0.19 & 0.14 & 0.17 & 0.12 & 0.19 & 0.18 & \\
\hline 7 & 0.41 & 0.33 & 0.38 & 0.26 & 0.41 & 0.31 & 0.10 & 0.25 & 0.28 & 0.23 & \\
\hline 8 & 0.25 & 0.31 & 0.24 & 0.16 & 0.30 & 0.22 & 0.20 & 0.40 & 0.27 & 0.36 & \\
\hline 9 & 0.36 & 0.26 & 0.37 & 0.23 & 0.27 & 0.35 & 0.20 & 0.64 & 0.18 & 0.18 & \\
\hline 10 & 0.19 & 0.19 & 0.21 & 0.21 & 0.19 & 0.19 & 0.18 & 0.17 & 0.13 & 0.12 & \\
\hline 11 & 0.41 & 0.41 & 0.39 & 0.24 & 0.41 & 0.26 & 0.18 & 0.12 & 0.35 & 0.38 & \\
\hline 12 & 0.47 & 0.33 & 0.44 & 0.32 & 0.43 & 0.41 & 0.14 & 0.57 & 0.25 & 0.14 & \\
\hline 13 & 0.32 & 0.31 & 0.35 & 0.23 & 0.32 & 0.37 & 0.27 & 0.71 & 0.26 & 0.36 & \\
\hline Avge $\Delta E$ & 0.29 & 0.26 & 0.28 & 0.20 & 0.27 & 0.25 & 0.16 & 0.34 & 0.20 & 0.21 & \\
\hline $\begin{array}{l}\text { Colorant } \\
\text { combination }\end{array}$ & 11 & 12 & 13 & 14 & 15 & 16 & 17 & 18 & 19 & 20 & Row $\Delta E$ \\
\hline 1 & 0.11 & 0.12 & 0.07 & 0.09 & 0.07 & 0.22 & 0.13 & 0.12 & 0.09 & 0.19 & 0.14 \\
\hline 2 & 0.08 & 0.08 & 0.14 & 0.12 & 0.18 & 0.28 & 0.23 & 0.20 & 0.24 & 0.22 & 0.19 \\
\hline 3 & 0.21 & 0.19 & 0.10 & 0.13 & 0.17 & 0.26 & 0.06 & 0.06 & 0.08 & 0.16 & 0.15 \\
\hline 4 & 0.19 & 0.20 & 0.21 & 0.21 & 0.11 & 0.48 & 0.22 & 0.21 & 0.25 & 0.29 & 0.23 \\
\hline 5 & 0.14 & 0.13 & 0.05 & 0.06 & 0.21 & 0.13 & 0.15 & 0.12 & 0.11 & 0.16 & 0.16 \\
\hline 6 & 0.15 & 0.14 & 0.13 & 0.13 & 0.15 & 0.15 & 0.16 & 0.13 & 0.18 & 0.16 & 0.17 \\
\hline 7 & 0.25 & 0.22 & 0.13 & 0.12 & 0.33 & 0.25 & 0.26 & 0.21 & 0.25 & 0.25 & 0.26 \\
\hline 8 & 0.30 & 0.29 & 0.17 & 0.22 & 0.17 & 0.47 & 0.14 & 0.15 & 0.12 & 0.32 & 0.25 \\
\hline 9 & 0.13 & 0.14 & 0.18 & 0.17 & 0.22 & 0.48 & 0.34 & 0.31 & 0.31 & 0.38 & 0.28 \\
\hline 10 & 0.13 & 0.13 & 0.17 & 0.16 & 0.11 & 0.27 & 0.14 & 0.14 & 0.19 & 0.17 & 0.17 \\
\hline 11 & 0.33 & 0.31 & 0.17 & 0.20 & 0.31 & 0.33 & 0.19 & 0.15 & 0.20 & 0.28 & 0.28 \\
\hline 12 & 0.19 & 0.17 & 0.13 & 0.09 & 0.37 & 0.34 & 0.36 & 0.31 & 0.32 & 0.34 & 0.31 \\
\hline 13 & 0.29 & 0.30 & 0.26 & 0.28 & 0.15 & 0.69 & 0.33 & 0.32 & 0.21 & 0.47 & 0.34 \\
\hline Avge $\Delta E$ & 0.19 & 0.19 & 0.15 & 0.15 & 0.20 & 0.33 & 0.21 & 0.19 & 0.20 & 0.26 & 0.23 \\
\hline
\end{tabular}

alter recipes manually in the Matchmaker system. The sample-standard $\Delta E$ values produced by the match prediction system were then compared with those used in the matrix method ( $\triangle E 0.5$ for row 1 and $\triangle E 1.0$ for row 2 , Table 8).

Table 8 row 1 shows the sample-standard colour difference from the Matchmaker system for each of 13 combinations predicted to be $\Delta E 0.5$ by the matrix method. Row 2 shows Matchmaker values predicted by matrix calculation to be $\Delta E$ 1.0. The results in Table 8 show close correlation between the two methods, and confirm the validity of the conclusions based on the matrix method.

\section{CONCLUSIONS}

1. It is possible to predict sensitivity of recipes to colour change.

2. Colour shows almost a linear change with concentration change along all semi-axes, for small concentration changes.

3. A matrix method can be used to calculate potential colour differences from colorant concentration changes.
4. A match prediction program can generate the following matrix coefficients:

$$
\begin{array}{lll}
R_{\mathrm{l} 1} & R_{\mathrm{ch} 1} & R_{\mathrm{h} 1} \\
R_{\mathrm{L} 2} & R_{\mathrm{ch} 2} & R_{\mathrm{h} 2} \\
R_{\mathrm{l} 3} & R_{\mathrm{ch} 3} & R_{\mathrm{h} 3}
\end{array}
$$

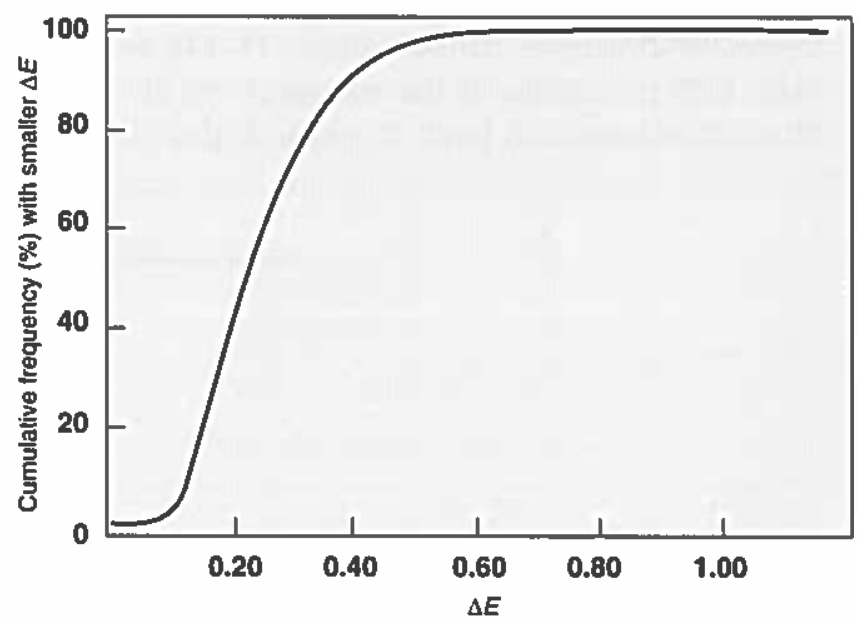

Figure 2 Effect on resulting colour with dye standardisation of $\pm 2.5 \%$ 


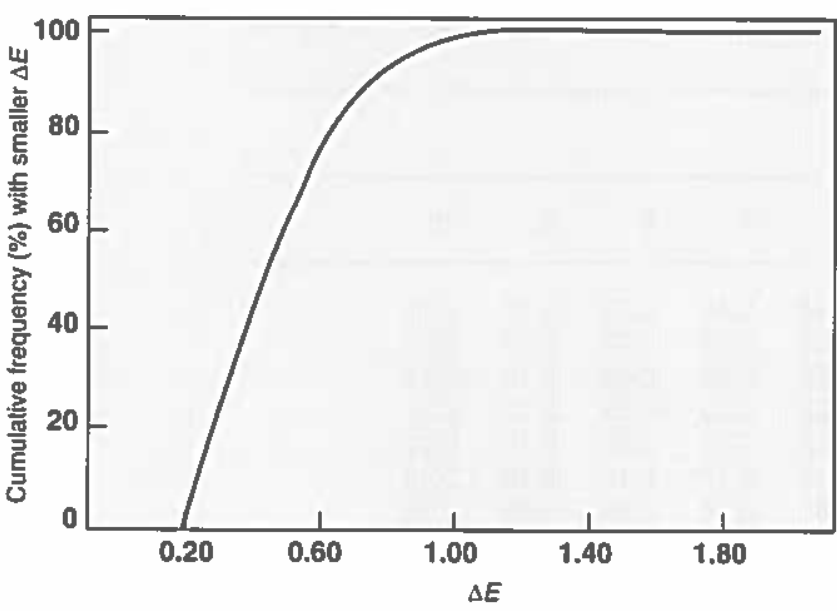

Figure 3 Effect on resulting colour with dye standardisation of $\pm 5 \%$

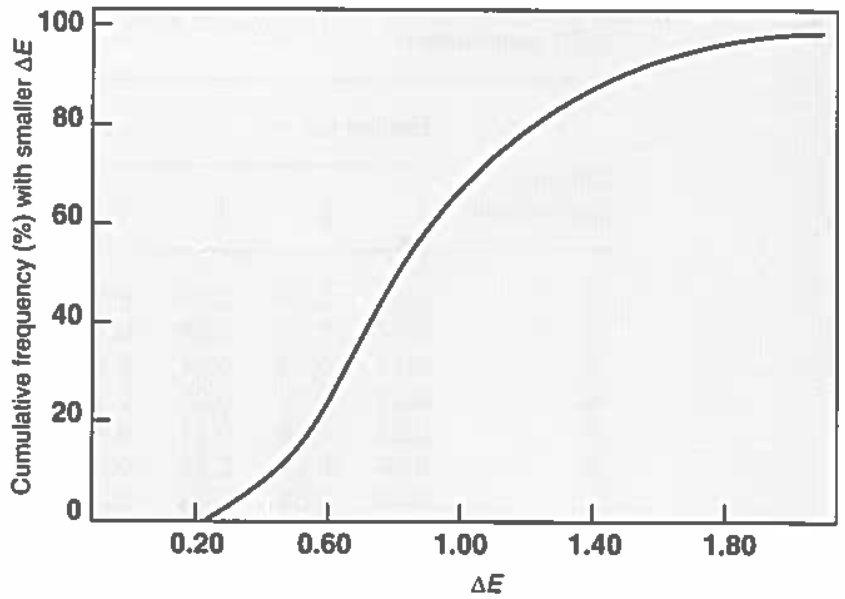

Figure 4 Effect on resulting colour with dye standardisation of $\pm 10 \%$

Table $8 \Delta E$ values by standard match prediction for recipe $20^{\mathrm{a}}$

\begin{tabular}{lllllllllllllll}
\hline $\mathrm{C}$ & 1 & 2 & 3 & 4 & 5 & 6 & 7 & 8 & 9 & 10 & 11 & 12 & 13 & Avge \\
\hline$\Delta E$ & 0.49 & 0.46 & 0.49 & 0.45 & 0.46 & 0.51 & 0.52 & 0.50 & 0.48 & 0.46 & 0.55 & 0.45 & 0.49 & 0.49 \\
$\Delta E$ & 0.91 & 0.88 & 0.99 & 0.89 & 0.92 & 1.03 & 1.02 & 0.99 & 0.95 & 0.89 & 1.08 & 0.95 & 0.94 & 0.96 \\
\hline
\end{tabular}

a For key see Table 6

by systematic alteration of recipe concentration. The 13 combinations of plus and minus movements described are sufficient for a three-colorant recipe. The full matrix method, involving synthetic reflectance curve generation is thus unnecessary for general use in calculating recipe sensitivity.

5. Average shade uncertainties of $0.23,0.46$ and $0.92 \Delta E$ units at $\pm 2.5 \%, \pm 5 \%$ and $10 \%$ colorant strength standardisations respectively would be expected.

6. From cumulative frequency analysis (based on 260 recipes), $98 \%$ of all coloured samples are within $\Delta E 0.5$ (attributable to colorant strength variation) with $\pm 2.5 \%$ colorant standardisation. The figures for $5 \%$ and $10 \%$ are $\Delta E 1.0$ and 2.0 respectively. For target shade $\mathrm{D}$, recipe 8 was twice as sensitive as recipe 7 .

7. High sensitivity is shown in worst-case scenarios, with colorants moving to their standardisation limits in opposite directions (combinations 11-13), an event with 0.23 probability if the colorants are at or near their standardisation limit. In recipe 8 , this combines with general sensitivity to produce a $\Delta E$ of 0.71 with strength changes of $\pm 2.5 \%$ (combination 13 ).

8. Given that colorant standardisation $\pm 2.5 \%$ alone can produce an average $\Delta E$ uncertainty of 0.25 , it is important to keep dosing accuracy as high as possible. Dosing to $\pm 0.5 \%$ would add a further uncertainty of $\triangle E 0.05$ to the average.

9. It would be relatively easy to modify match prediction programs to test individual recipe sensitivity to possible colorant strength variations, and provide practical warning of sensitive combinations.

\section{REFERENCES}

1. J Park, /.S.D.C., 107 (1991) 193

2. H H Sumner, J.S.D.C., 92 (1976) 84

3. R A Nickson and H H Sumner, J.S.D. C., 92 (1978) 493.

4. D P Oulton and P Chen, J. China Text. Univ., 4 (1993) 81

5. B Glover, J.S.D.C., 107 (1991) 184

6. CJ Hawkyard and D P Oulton, J.S. D.C., 108 (1992) 309.

7. Macbeth Matchmaker Manual, 1987. 